\title{
ÜBER DIE PARALLEILSTELLEN IN DER ANGELSÄCHSISCHEN POESIE.
}

Sarrazin hat in seinen Beowulfstudien zwischen Beowulf einerseits und Cynewulf's echten und den ihm zugeschriebenen dichtungen andererseits, ferner zwischen B.-Cynewulf einerseits und der tubrigen angelsächsischen poesie andererseits eine grosse anzahl parallelstellen nachgewiesen. Nur in Ps., Hy., Met., Fæ. und Zaub. sollen sich keine ubereinstimmungen mit B.-Cynew. finden. Es schien mir auffallend, dass die erwähnten gedichte allein eine ausnahme bilden sollten. An das von Sarrazin gesammelte material anschliessend, untersuchte ich: 1) Ob Ps., Hy., Met., Fæ., Zaub. nicht vielleicht doch anklänge an B.-Cynew. enthielten. 2) $\mathrm{Ob}$ parallèlstellen nur zwischen Cynew. und der ubrigen poesie sich fänden, oder ob etwa auch nichtcynewulfische dichtungen untereinander gemeinsamkeit des ausdrucks zeigten. Schliesslich, als diese vermutangen sich bestätigten, 3) ob sich etwas uber die herkunft und bewegung der parallelstellen ermitteln liesse. Im laufe der arbeit fanden sich noch einige nachträge zu Sarrazin kap.IV. Ich fuhre zunächst die stellen an, behaupte aber keineswegs, unbedingte vollständigkeit erreicht $\mathrm{zu}$ haben, obwol ich dieselbe stets angestrebt. Im gegenteil, ich bin tuberzeugt, dass sich sowol zu Sarrazin's als zu meinen belegstellen noch immer nachträge finden werden. Doch glaube ich, dass das jetzt vorhandene material hinreicht, um daraus ein urteil uber die bedeutung der parallelstellen fällen $\mathrm{zu}$ können.

Bei den folgenden aufzählungen gehe ich von den nach S. (s. 153) Cynewulf'schen werken aus und vergleiche mit ihnen die tubrigen ags. dichtnngen in der reihenfolge von Grein's Bibliothek der ags. Poesie. Die versschltisse, anf deren thereinstimmung $\mathrm{S}$. besonderes gewicht legt, bezeichne ich, wo sie nicht ohnehin ersichtlich sind, durch einen senkrechten strich. 


\section{Nachtrag zu Sarrazin's material.}

B. 8 weox under wolcnum

n9 æghwilc para ymbsittendra

$n$ ofer hronrade

n 100 orpæt an ongan

fyrene fremman

n 119 sorge ne curon !

n 301 flota stille bad ।

n916 Hwilum fealwe stræte

mearum mæton

n $93 \quad$ swa wæter bebugeঠ

n 250 næfne him his wlite leoge

${ }_{n} 654$ and pæt word acwæð।

${ }_{n} 658$ hafa nu and geheald

„ 983 ofer heahne hrof

„ 1082 pæt he ne meahte on pam meðelstede

n 1277 gifre and gealgmod

n 1569 secg weorce gefeah |

n 2320 ær dæges hwile |

" 2696 swa him gecynde wæs ।

¿2805 heah hlifian

n 3035 pa wæs endedæg ।

n 145 orpæt idel stod

husa selest

n 547 nipende niht

n 2870 ower feor orre neah findan meahte

n 547 nipende niht

n955 awa to ealdre

¿ 1736 adl ne yldo

" 99 Swa pa drihtguman dreamım lifdon

eadiglice oðpæt an ongan fyrene fremman

'n 1024 Beowulf gepah ful on flette
Gen. 1702 weox pa under wolcnum

n 2488 anra gehwilcum jmbstandendra

n 205 geond hronrade

n 30 pe pone unræd ongan ærest fremman

n 19 firena fremman

n 18 Synns ne cuŏon ।

Exod.550 here stille bad।

n 170 Hwilum of pam werode wlance pegnas

mæton milpaðas meara bogum

Dan. 321 swa heofonsteorran bebugat

n 416 nales me sefa leoge 283 and pa word acwæठ।

198 habban ne healdan

442 on heahne hrof

n 145 ne meahte pa seo menigéo on pam merelstede

n 230 grim and gealhmod

„268 para pe py weorce gefegon|

n 349 on dæges hwile |

"3 swa him gecynde wæa |

n 603 heah hlifian

n 679 pa wæs ondedæg ।

Wand. 86 otpæt ...

eald ents geweorc idlu stodon

n 104 niped nahtscua

„ 26 hwær ic feor oððe neah findan meahte.

Seef. 31 nap nahtscua

$n 79$ awa to ealdre

$n$ io adl oppe yldo

Rui. 22 Beorht wæron burgræced, burnsele monige, heah horngestreon, hereweg micel

meodoheall monig, mandreama full, otpæt pæt onwende Wyrd seo swite

Wids. 3 oft he on flette gepeah mynelicne maddum 
B. 1041 næfre on ore læg wiðcures wig ponne...

n 343 Beowulf is min nama

n656 siððan ic hond and rond hebhan mihte

n 658 hafa nu and geheald

n 675 gespræc gylpworda sum

n 2017 folca flet eal geondhwearf |

2030 lytle hwile ।

, 754 forht on ferhðe; no py ær fram meahte

n 1337 he at wige gecrang |

„1545 hyre seax geteah

brad and brunecg

, 1573 wæpen hafenade

heard be hiltum Higelaces

pegn

yrre and anræd

n 2524 Nelle ic beorges weard, feond oferfleon fotes trem

n $2646 \quad \mathrm{Nu}$ is se dæg comen

2984 wælstowe wealden moston

1950 ofer fealone flod

464 ofer yoa gewealc

1861 ofer ganotes bæð

14 pone god sende

folce to frofre

n 1898 mearum and maðmum

2861 pam pe ar his elne forleas.

471 ofer wæteres hrycg |

730 his mod ahlog |

1622 pas lænan gescerft

El. 109 byman sungon |

731 ealne gehwyrft and uprador

ᄁ530 sevde soðcwiðum

^ 924 goda geasne

„983 ofer heahne holm

134 flugon on fæsten and feore burgon

n 88 fæle friðowebba

239 ofer eargeblond

74 ær оððe sið

„292 wæron wuldorcyninge drihtne dyre
Wids.119 ful oft pær wig ne alæg ponne...

Fi. 24 Sigeferठ is min nama

By. 235 pa hwile pe he wæpen mæge 1

habban and healdan, heardne mece

gar and god swurd

By. 274 gylpwordum spræc

Sal. 185 land eal geondhwearf |

„367 lytle hwile ।

By. 317 Ic eom frod feores; fram ic ne wille

" 324 he on hilde gecranc ।

^ 162 bræd bill of sceðe

brad and brunecg

" 42 bord hatenode

wand wacne æsc, wordum mælde

yrre and anræd

„247 pæt ic heonan nelle

fleon fotes trymm

" 104 wæs seo tid cumen ।

n 95 wælstowe wealdan mote।

Aepelst. 36 on fealone flod

Eadg. 45 ofer yoz gewealc

Eadg. 46 ofer ganotes bæర

Men. 228 fæder engla

his sunu sende

folcum to frofre

Gn.Ex. 88 mearum and marmum

n 188 pæt he elne forleose

Sal. 19 on wæteres hricg |

"178 his ferht ahlog ।

n 326 on pisse lænan gesceafte

Exod.160 byman sungon | (Dan.192)

n 26 eorðan ymbhwyrft and uprodor

Dan. 446 sevton soðcwidum

Wal. 46 goda geasne

Wand. 82 ofer heahne holm

Wald. 15 on weal fleon, lice beorgan

Wids. 6 fælre friððowebban

Aepelst. 26 ofer eargeblond

Men. 200 sið oбre ær

" 192 wæron

drỵhtne dyre 
El. 1189 be pam se witga sang |

n 470 asced para scylda

Jul.417 bifolen in foldan

n 564 pæt fyr tosceaf ।

566 pone lig towearp

n 335 oбre feor oðde neah fundne weorðen

„ 495 peah ic gesitte sumorlangne dæg

n 385 bugan from beaduwe

n 526 ic behlihhan ne pearf।

n 547 ic to sode wat

An.1506 ea inflede

n 1130 freonda feasceaft

ๆ 37 pæt hie ne murndon æfter mandreame

n 1180 ponne gemet wære |

n 1268 acol for py egesan

" 1456 man on moldan

n 1012 Hiht wæs geniwad |

n 368 ofer yoa gepring ।

n 818 hreðre behabban

274 ofer hwæles eðel

403 æt lides stefnan |

457 for frean egesan ।

564 he wundra fela wordum gecyбte

sweotulra and gesynra

n 293 ofer fisces bæठ।

๓ 42 manfulra hloঠ।

Gû. 1262 scadu swetredon

n 543 pone grimman gryre

n 664 acol for pam egesan

n 130 dygle stowe

n 210 hie gielpan ne porfton |

n 507 cuð is wiðe

Cri.187 clæne

womma lease

n 394 wide and side ।

469 geond woruld innan

830 lifdon leahtrum $\mathrm{fa}$ ।

1015 for fæder egesan |

686 geofum unhneawum |

785 us secgar bec ।

547 swa gewritu secgað।

468 swa ar beforan sungon | witgens word

Rä. $81^{24}$ gifrost and grædgost
-Men. 59 swa se witga sang ।

Sal. 56 ascesठan of scyldum

Dan. 560 foldan befolen

n 340 pone lig toscesf |

Wand. 26 hwær ic feor oðte neah findan meahte

Kl. 37 pær ic sittan mot sumorlangne dæg

By. 185 bugon fram beaduwe

Aepelst. 47 hlihhan ne porftan ।

Sal. 429 ic to sozum wat

Gen. 232 ea inflede

n 2699 freonda feasceaft

Dan. 571 pu ne gemyndgast after mandreame

n 250, 492 ponne gemet wære |

n 726 acol for pam ugesan

n 567 man on moldan

Kr. 148 Hiht wæs geniwad |

Sch. 79 - under wætera gepring |

n 10 behabban on hreðre

Seef. 60 ofer hwæles edel (Eadg. 48)

n 7 mt nacan stefnan ।

n 101 for godes egesan ।

Men. 126 hæfdon mænige ær wundra geworhte swutelra and gesynra

Run. 16 ofer fisces bæð ।

Sal. 148 manfulra heap |

Gen. 133 sceado sweart swiðrien

Dan. 439 of pam grimman gryre

n 726 acol for pam egesan

Pa. 37 dygle stowe

Aepelst. 44 gylpan ne porfte |

Gn. Ex. 199 cuð wæs wide

Dom. 93

clæne

womma lease

" 10 wide and side ।

Pa. 4 geond worold innan

Wal. 66 pæt he bið leahtrum fah |

Seef. 101 for godes egesan ।

Wids.139 geofum anhneawne |

Aepelst. 68 pæs pe us secgað bec ।

Eadg. 14 pæs pe gewritu secgað।

Men. 59 swa se witga sang ।

Seef. 62 gifre and gredig 


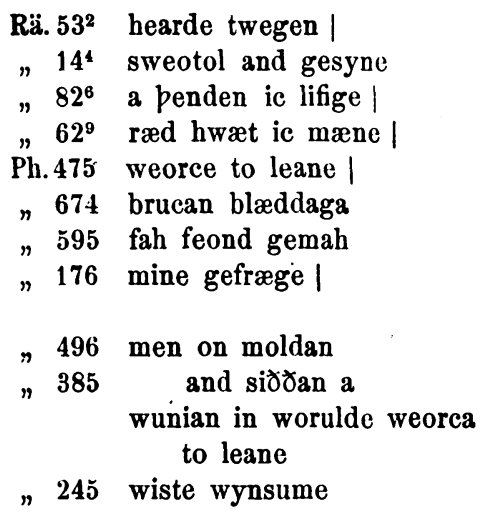
By. 80 modige twegen |
Men. 128. sweotolra and gesynra
Fï. 8 a penden pu lifige ।
Sal. 236 saga hwæt ic mæne ।
Gen. 37 weorce to leane |
n 200 brucað blæddaga
Wal. 39 flah feond gemah
Wids. 71 mine gefræge $\mid$ (Eadg. 9, 36, Men. 2i)
Eadg. 25 men on moldan
Men. 146 hæfo nu lif wið pan mid wuldorfæder weorca to leane
Leas. 22 wynsume wist.

\section{Parallelstellen zwischen den nach Sarrazin} Cynewulfischen dichtungen einerseits, und Ps., Hy., Met., Fæd., Zaub. andererseits.

B. 801 sawle secean

"9 oðpæt him æghwilc para ymbsittendra ofer hronrade hyran scolde

„80 he beot neh aleh

n 259 wordhord onleac ।

"561 swa hit gedefe wæs |

" 576 under heofenes hwealf

$" 687$ swa him gemet pince |

" 1119 wand to wolcnum

, 2182 ginfæstan gife

, 1609 ponne forstes bend fæder onlæteð।

n 1622 pas lænan gesceaft |

" 1754 lichoma læne

n 1224 penden pu lifige |

n407 Wes pu Hropgar hal

n 471 ofer wæteres hrycg

El. 951 pær awa scealt wiðerhy cgende wergðu dreogan

n 239 ofer eargeblond

, 1024 golde beweorcan and gimcynnum
Ps. $141^{5}$ mine sawle secan wolde

Met. $9^{14}$ eal pæt Nerone nede oððe lustum

heaঠorinca gehwilc heran scoolde.

$n 1^{30}$ he pæt eal aleag

$" 6^{1}$ wordhord onleac !

n $26^{98}$ swa hit gedefe ne wæas |

" $10^{7}$ heofenes hwealfe

n 2942 swa him gemet pinced।

"31 ${ }^{13}$ windar ofer wolcnum

" $20^{237}$ ginfæsta gifa

" $11^{27}$ ponne him sigora weard his gewealdleðer wille onlætan |

" $20^{157}$ pas lænan gesceaft |

, 26113 lichoman lænne

$26^{106}$ lænes lichoman

Fæ. 8 penden pu lifge ।

Zaub. $1^{\text {67 }}$ Hal wes pu folde

" $4^{28}$ ofer sæs hrycg

Ps. 68 ${ }^{2}$ on sæs hricg

Hy. $4^{57}$ forpon hy longe sculan

werge wihte wræce prowian

Met. $8^{30}$ ofer eargeblond

$n$ 154 golde geglengde and gimcynnum 
KAII,

Jul.112 heofon and eoroan and holma begong

n 168 ginfæsto gife

n 295 heafde beheawan

Cri.394 wide and side ।

n 785 us secgat bec ।

n 204 leoman onlyhte

n 382 earme eoroware

n 667 singan and secgan

, 677 ofer sealtne $8 \circledast$

n 693 hædre heofontungol

n 910 eadgum and earmum

Rï. $35^{\circ}$ blowan and growan |

n $14^{4}$ sweotol and gesyne

Ân.1215 herd hyge pinne, heortan staðola

n 1284 pu wæst and const |

" 1558 feasceaft hæle $ठ$

n 61 grette gumens brego geomran stefne

... and pus wordum cwæठ

n 195 holma begang ।

" 1628 mine gefrege ।

Gu.1059 mode and mægne ( $\mathrm{Ph} .471)$

„543 in pone grimman gryre

n 761 halig hyrde heofonrices weard I

Gen.1744 pa se halgs spræc heofonrices weard |

$\mathrm{Ph} .117$ siðбan wuldres gim ofer geofones gong grund geciner

n 42 ealne middangeard
Met. $25^{6}$ golde gegerede and gimcynnum

n $11^{30}$ heofon and eortan and eal holma begong

n $20^{227}$ ginfæsta gifa

$n 1^{43}$ heafde beheawan

Ps. 56 6. 13 wide and side ।

Hy. $7^{20}$ pæs pe secgat bec ।

Met. $2^{36}$ leoman onlihtan

$n 4^{57}$ earme eorowaran

" $2^{17}$ secgan oб $\theta$ singan

, $19^{16}$ on sealtne sæ

n 22: hador heofontungol

Zaub. $4^{41}$ earmum and eadigum

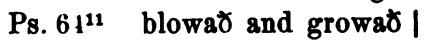

Hy. $9^{50}$ swutele and gesyne

Ps. $7^{10}$ næfdon heora heortan hige gestaðyelod

${ }_{n} 55^{8}$ ic wat and can ।

Hy. $4^{112}$ feasceaft hæle

Met. $1^{83}$ cleopode to drihtne geomran stefne gyddode pus

$n 1^{80}$ eal holma begong $\mid$

n 2082. 248 mine gefræge।

" $4^{27}$ mode and mægne

Zaub. $8^{3}$ wip pane grimman gryre

" $1^{27}$ pone haligan heofonrices weard ।

Met. $22^{28}$ ponne swegles gim hador heofontungol hlutrost scineð

Zaub. ${ }^{4}$ geond ealne middangeard.

\section{Parallelstellen $z$ wischen den nach Sarrazin nichtcynewulfischen gedichten.}

Gen. 346 wio god winnan।

„ 537 his word ... wel healdan lestan bis lare

n. 1180 land and leodward

n 2737 he wrs leof gode

, 2909 Here stille gebad।

^75 susl prowedon ।

ๆ 962 eard and eðel

1102 mid grimme gryre
Exod.514 hie wið god wunnon ।

„560 gif ge gehealda halige lare

n 57 land and leodweard

n 12 He wæs leof gode

n 300 mere still bad |

Dan. 621 susl prowode ।

n 612 eard and eðel

n 439 of pam grimman gryre 
Gen. 1669 pa foremeahtigan folces ræswan

" 2332 lufan and lisse

n 1820 geseah Egypta

hornsele hwite and hea byrig

beorhte blican

^ 1970 blachleor ides

n 1991 Handum brugdon

hæleð of scæðum hringmæled sweord

ecgum dihtig

n 76 pystrum bepeahte

" 104 pes wide grund

stod deop and dim drihtne fremde

idel and unnyt

n 2005 weold wælstowe

n 2723 pær pe leofost sie |

n 1332 on lides bosme

1990 wælgara wrixl

1999 Gewiton feorh heora

fram pam folcstede fleam nergan

n 2057 heardan handplegan

๓ 2192 ofer brad brimu

„ 2772 swa him cynde wæron æðelo fram yldrum

n 17 demdon drihtnes

n 125 swa se wyrhta bebead |

, 134 geond sidne grund।

375 ufan and neoðone

418 windan on wolcne

962 eard and eðol

1517 eorte algrene

1696 purh his mihta sped

" 2596 wunode wealdende leof

dægrimes worn and his doh-

" 1051 wineleas wrecca [tor twa

„2027 Aner and Manre Escol priddan $\mid$

" 2723 par pe leofast sie

Exod. 160 byman sungon

n 546 duguð on dreame drihten heriad

" 301 Hofon herecyste hwite linde

segnas on sande
Dan. 667 frod foremihtig folces ræswa

ก 340 mid lufan and lisse

Jud. 136 pæt hie sweotollice geseon mihten

pære wlitegan byrig weallas blican

n 128 blachleor ides

n 229 mundum brugdon

scealcas of sceatum scir-

mæled swyrd

ecgum gecoste

Hö. 55 bepeahte mid pystre

Wand. 86 orpæt burgwara breahtma lease

eald enta geweorc idlu stodon

By. 95 wælstowe wealdan

, 23 pær him leofost wæs ।

Aepelst. 27 on lides bosme

n 51 wæpengewrixles

n 36 on fealone flod feorh generede

" 25 heardes hondplegan

n 71 ofer brade brimu

n 7 swa him geæठelo wæs fram cneomagum

Ap. 10 deman drihtnes $æ$

Leas. 39 swa him god bebead ।

Met. $20^{127}$ geond pisne sidan grund।

n $20^{141}$ ufane and neotane

n $31^{18}$ windat ofer wolcnum

n $24^{50}$ eard and erel

n $20^{78}$ eorठe ælgreno

n $4^{9} \quad$ purh pinra meahta sped

n 2638 pær Apollines

dohtor wunode dægrimes

Hy. $4^{90}$ wineleas wrecca [worn

\# $7^{95}$ beofonwaru and eorðwaru helwaru pridde I

Ps. $80^{12}$ swa him leofust wæs

Dan. 192 byman sungon!

n 257 ofstum heredon drihten on dreame

By. 244 Leofsunu gemælde and linde ahof bord to gebeorge 
Exod. 481 fæge crungon |

$n 129$ fus on foroweg

n 80 wand ofer wolcnum

n 346 morgen mæretorht

Dan. 327 hat and ceald

n 198 habban ne healdan

223 bitera dear

442 on healme hrof heofona rices

613 for pam gilpe

» 335 metudes miltse and his mihta sped

Jud. 205 pæs se hlanca gefeah wulf in walde and se wanna hrefn

wælgilfre fugl ...

earn ætes georn urigferera

salowigpada . . .

hyrnednebba

Hö. 2 gumena gemot |

Dom. 68 no pæs gilpan pearf |

Seel. 81 feldgangende feoh butan snyttro

Mod. 14 wlonce wigsmiðas

Seef. 46 ymb yða gewalc |

„60 ofer hwæles erel

Bo. 13 pæt pu sinchroden sylf gemunde

on gewitlocan wordbeotunga

pe git on ærdagum oft gespræcon

penden git moston on meodoburgum

eard weardigan, an lond bugan

freondscipe fremman

Wids. 1 wordhord onleac ।

Fi. 29 celod bord

By. 169 har hilderinc

n 283 clufon cellod bord.

" 293 on wælstowe wundum sweltan

"23 pær him leofost wæs

"98 ofer scir wæter scildas wegon

„70 ne mihte ... derian
Aepelst. 10 hettend crungon

Men. 218 fus on fortweg

Met. $31^{12}$ windar ofer wolcnum

n $13^{61}$ morgen meretorhtne

Dom. 106 hates and cealdes

By. 236 habban and healdan

Eadw. 26 dear se bitera

Met. 24 ${ }^{3}$ ofer heane hrof heofones pisses

n einl. 8 for his gilpe

Sal. 492 metodes miltse and his mæga ræd

Aepelst. 61 salowigpadan pone

sweartan brefn

hyrnednebban and pone heasupadan

earn ...

. . . and pæt græge deor wulf on wealde

Aepelst. 50 gumena gemotes |

" 44 gilpan ne porfte ।

Sal. 23 feldgangende feoh butan gewitto

Aepelst. 72 wlance wigsmiðas

Eadg. 45 ofer yða gewealc ।

n 48 ofer hwæles exel

By. 212 Gemun pa mæla pa we oft æt meodo spræcon ponne we on bence beot ahofon

hæleঠ on healle ymbe heard gewinn

Met. $6^{1}$ wordhord onleac ।

By. 283 cellod bord

Aepelst. 39 har hilderinc

5 bordweall clufon

n 43 on wealstowe wundum forgrunden

Ps. $80^{12}$ swa him leofust wæs

Met. $1^{2}$ eastan of Sciððia sceldas læddon

Zaub. $6^{16}$ derian ne moste 
By: 80 Aelfre and Marcus modige twegen

„42 Byrhtnoð maðelode, bord hafenode

wand wacne æse, wordum mælde

yrre and anræd, ageaf him andsware

Aepelst. 68 pæs pe us secgad bec

$n \quad 13 \quad$ siððan sunne upp on morgentid mære tungol glad ofer grundas godes candel beorht

op pæt seo æpele gesceaft sah to setle

Aepelst. 68 pæs pe us secgað bec

ealde uðwitan

' $\quad 26$ ofer eargeblond

n 53 gewiton him pa ofer deop wæter Dyflin secan

Eadg. 22 ceas him oder leoht wlitig and winsum

Men. 57 mannum to frofre ।

" 129 swutelra and gesynra

^ 162 betwux wife and were wurde acenned

n 87 be fæder leafe

n 88 bring $\delta .$. sumor to tune wearme gewideru

, 59 swa se witega sang

Ap. 84 ealle forhogodan ।

Leas. 2 manig and mislic

Kr. 16 gegyred mid golde, gimmas hæfdon

Gn. C 23 steap and geap

Ps. 64" blowar and growad।

"682 on sæs hricg

Met. 5 ${ }^{\text {44 }}$ pæt hit seo ece ne mot innan geondscinan sunne
Zaub. $4^{37}$ Fille and Finule, fela mihtigu twa

Wald. 43 Waldere maðelode wiga ellenrof

hæfde him on handa hildefrofre

gưbilla gripe, gyddode pus

Eadg. 14 pæs pe gewritu secgað

Men. 109 on pam gim astih $\delta$

on heofones up hyhst on geare

tungla torhtast and of tille agrynt,

to sete sigeð

Hy. $7^{20}$

Met. $22^{53}$

pæs pe secgat bec

pæt us reahte gio eald uðwita

n $8^{30}$ ofer eargeblond

Sal. 203 gif pu gewitest on Wendelese

ofer Coferflod cyððe secan

Ap. 19 him ece geceas

langsumre lif, leoht unhwilen

Hy. $7^{83}$ mannum to frofre |

"950 swutele and gesyne

$" 7^{87}$ pe fram wife and were wurdon acenned

Met. $11^{\text {o7 }}$ butan frean leafe

$n \quad 11^{80} \quad$ sumor of cymed wearm gewideru

Zaub. $1^{85}$ swa se witega cwæठ

Ps. $77^{82}$ eal forhogode

Met. $25^{8}$ manegum and mislicum

$" 25^{6}$ golde gegerede and gimcynnum

Sal. 413 steap and geap

Met. $20^{90}$ bloweð and groweð

Zaub. $4^{28}$ ofer sæs hrycg

Sal. 338 ne mot seon sunne siðe gesceafte scire geondscinan

Aus dem vorgeftuhrten material ergeben sich für die von Sarrazin entwickelte theorie folgende konsequenzen:

1. Aus den unter 1 angegebenen stellen folgt, dass die 
abhängigkeit der einzelnen dichter von Cynew. eine noch weit grössiere war, als S. sie darstellt.

2. Es mussten auch Ps., Hy., Met., Fæ., Zaub. von Cynew. oder einem seiner nachahmer herrthren. Die ganze ags. poesie wäre somit unter dem einflusse dieses einzigen dichters entstanden.

3. Einige gedichte haben unter einander parallelstellen gemein, welche im Cynew. nicht zu belegen sind (Mod. 14 : Aep. 72, Seel. 81 : Sal. 23, Men. 90 : Met. $11^{61}$, Met. $11^{44}$ : Sal. 338, Gen. 2596 : Met. 26 ${ }^{33}$ ). Dieselben mussten also aus verloren gegangenen werken Cynew.'s entlehnt sein, oder, wie S. (s. 187) vermutet, aus anderen nicht erbaltenen mustern des epischen stiles stammen. Es gäbe dann keine spur eines selbständigen ags. stiles, alles wäre unter fremdem einfluss entstanden.

Durch diese konsequenzen wird der ags. poesie eine entwicklung zugeschrieben, wie sie in historischer zeit in keiner literatur nachzaweisen ist. Es ist doch nicht wahrscheinlich, dass sich in der ags. literatur allein erscheinungen finden, die nirgends anch nur ein entferntes analogon haben.

Sarrazin grttndet seine theorie auf die annahme, parallelstellen seien ein charakteristikon fur einen bestimmten autor. Diese hypothese spielt in der Cynewulffrage eine grosse rolle. Auf grundlage von thereinstimmungen mit seinen echten werken hat man Cynew. die verschiedensten ags. gedichte zugeschrieben. Wenn etwas eigenttumlichkeit eines gewissen dichters sein soll, so darf es anch nur bei ihm allein vorkommen. Zieht man nun aber. zu dem S.'schen material noch die verschiedenen einzeluntersuchungen (namentlich Charitius, Fritzsche Anglia II, Lefèvre Anglia VI, Rieger Zs. f. d. Ph. I u. a.) und die hier gegebenen belegstellen heran, so wird man zwischen irgend welchen, auch nur einigermassen umfangreichen ags. gedichten parallelstellen nachweisen können. Wenn nun letztere gemeingut der gesammten poesie sind, wie sollen sie fur einen bestimmten dichter charakteristisch sein? Wenn es wirklich parallelstellen gäbe, welche als ein derartiges charakteristikon gelten krnnten, so wtirden sich solche wol am ehesten mit hilfe der sicher Cynewulfischen dichtungen ermitteln lassen. In den letzteren wtirde es vielleicht gewisse phasen geben, welche sich vor denen der thbrigen gedichte in irgend einer weise auszoichnen. Es sind mir ausser den von Ramhorst, 
'Das altengl. Gedicht vom heil. Andreas' und von Hoffmann, 'Reimformeln im Westgermanischen' angefuhrten noch folgende parallelstellen zwischen den echt Cynewulfischen dichtungen aufgefallen:

Elene.

371 gedwolan fylgdon |

859 geprowade prymmes hyrde

979 pær hie hit for worulde wendan meahton

1050 Criste gecweme

1129 egesan geaclod

1161 het pa gefetigan

427 fæstlice ferhð staðelien

Elene.

188 to heofonum astag |

252 ald yðhofu oncrum fæste

279

418

673

737

808

on gemot cuman ।

giedda gearusnottor

godes gastsunu

mid pa leohtan gedryht

Nu ic purh soð hafn seolf gecnawen

on heardum hyge, pæt pu hælend eart

565

... heo wæron stearce stane heardran

1006 hæle $\gamma$ hwætmode to pære haligan byrg

1132 pa wæs wopes hring

1148 geornlice gastgerynum

\section{Crist.}

90 gehðum mænaठ।

481 geond ealne yrmenno grund |

662 monigfealde modes snyttru

1219

scyppend scinende scrife $\circlearrowright ~ b i$ gewyrhtum

eall æfter rihte

1580

penden him lic and gæst somodfæst seon

\section{Juliane.}

202 gedwolan fylgest |

448 geprowade prymmes ealdor

570 pær he hit for worulde wendan meahte

259 Criste gecweme

268 egesan geaclad

60 het pa gefetigan

270 fæstlice ferhð staðelian

Crist.

737 to heofonum astag ।

864 ealde yomearas ancrum freste

943 on gemot cuman |

713 giedda gearusnottor

660 godes gastsunu

515 mid pas engls gedryht

1187 Leode ne cuðan

modblinde men meotud oncnawan

flintum heardran .

461 hæle $\delta$ mid hlaford, to pære haligan byrg

537 pær wæs wopes hring

440 geornlice gastgerynum

$$
\text { Juliane. }
$$

391 gehбu mænan ।

10 ofer ealne yrmenne grund |

366 monigfealde modes gælsan

726 seo pryness ymbsittende

in annesse ælda cynne

purh pa sciran gesceaft scrife $\delta$

bi gewyrhtum

meorde monna gehwam

714 pendan gæst and lic geador siðedan

Es mögen sich vielleicht hie und da noch einige anklänge finden, aber jedenfalls werden sie night zahlreich und nur von untergeordneter bedeutung sein. Cynewulf's echte werke haben mit anderen gedichten weit mehr parallelstellen gemein als unter- 
einander. Hoffmann macht a. a. o. s. 40 dieselbe bemerkung bezttglich der reimformeln. Er findet deren eine grössere anzahl zwischen Gấlac und den Cynewulfischen dichtungen als die letzteren untereinander ausweisen. Was die genanigkeit der ubereinstimmung betrifft, so ist dieselbe nicht grösser als bei parallelstellen anderer gedichte. Es gibt also keinen unterschied zwischen den Cynewulfischen und den tubrigen phrasen.

Es ist wol das natlirlichste, die parallelstellen als einen gemeinsamen poetischen formelschatz zu betrachten, aus welchem alle ags. dichter unabhängig von einander je nach bedarf ihren ansdruck entnahmen. In diesem sinne mag man von einem gemeinschaftlichen muster sprechen, nach welchem alle ags. dichter arbeiteten oder ihren stil bildeten. Dieses muster ist aber nicht in einer bestimmten dichtung zu suchen, sondern dasselbe mulsste sich vielmehr mit hilfe der gesammten ags. poesie wenigstens annäherungsweise rekonstruieren lassen.

Woher hatten die Angelsachsen diesen formelvorrat?

Ein teil desselben lässt sich auch in der altsächsischen und althochdeutschen alliterationspoesie nachweisen. Sievers hat in seiner schrift: 'Der Heliand und die ags. Genesis' gemeinsamkeit des ausdrucks zwischen altsächsischer und angelsächsicher dichtung nachgewiesen und dann in seiner ausgabe des Heliand die den altsächsischen entsprechenden ags. bezeichnungen für einzelne begriffe zusammengestellt. Im folgenden zähle ich weitere parallelstellen auf zwischen Heliand und den ahd. alliterierenden dichtungen einerseits und der gesammten ags. poesie andererseits. Die stellen sind nach der reihenfolge im Heliand und in den ahd. dichtungen geordnet.

Heliand.

5 mid uuordun endi mid uuercun

41 himil endi ertha endi al that sea behlidan egun

51 managon te helpun

70 than lang hie geuusld ehta Erodes thes rikeas

559 sithor ik muosta thieses erlo folcas giuualdan theses uuidon rikeas ne saca ne sundea

354 is land is lindi
Ags. poesie.

B. 1833 wordum and weorcum

Met. $11^{80}$ heofon and eortan and eal holma begang

B. 1709 hæleðum to helpe

Cri. 427 gumum to helpe

B. 1859 penden ic wealde widan rices
Ph. 54 ne synu ne sacu
B. 2472 synn and sacu
Edw. 25 land and leode 
367 Thar gifran ik

415 uundun thuru thiu molkan

495 obar thesan middilgard

588 an thesan middilgard

554 Hueder ledeat gi uundan gold te gebu huilicon gumono? te hui gi sus an gange cumat gifaran an fathie? Huat, gi nethuanan ferran sind

erlos fan orron theodon. Ik gisiho that gi sind ediligiburdion

cunnies fan cnuosle guodon: nio hier er sulica cumana ni uurठun

eri fan odron thiodon sithor ik muosta thieses erlo folcas giuualdan theses uuidon rikeas. Gi sculon mi te uuarun seggian

far theson liudeo folca bihui gi sin te theson lande cuman.

734 ni uuarth sið noh err |

941 so mikilu is hie betera than ik !

968 so muarth im is hugi blið $i$

1293 is mur antloc

1393 unliti endi uunsam

1542 an thesaro lehnun uueroldi

1765 unerthan te unillien

1836 thea hie for thero menigi gisprak 1

2005 Uuerod blithoda

uuarun thar an luston liudi atsamna

2009 uuas thar erlo drom |

2254 nis nu lang te thiu

2781 tho ni was lang te thiu

2071 Thuo uuarth that so uuido cuth

2076 Ne mag that gitelliean man geseggian to suothen

2603 thar sculun sia gibundana bittra logna

thraunerc tholon

Anglia, XII. band.
B. 2773 pa ic . gefrægn

Exod. 80 wand ofer wolcnum

B. 75 geond pisne middangeard

(Wand. 75)

Kr. 104 on pysne middangeard

B. 237 Hwæt syndon ge ... pe pus brontne ceol [mon ofer lagustræte lædan cwohider ofer holmas hringedstefnan?

Ic wæs endesæta, ægwearde heold ...

no her cuðlicor cuman ongunnon

lindhæbbende ... Næfre ic maran geseah

eorla ofer eorpan ðonne is eower sum,

secg on searwum: nis pæt seldguma

wæpnum geweorðad, næfne him his wlite leoge

ænlic ansyn! Nu ic eower sceal [is selost

frumcyn witan ... ofost

to gecyðanne, hwanan eowre cyme syndon.

El. 240 ne sið ne ær ।

B. 469 se wæs betera ðonne ic।

Dan. 117 Næs him bliðe hige

B. 259 wordhord onleac /(Wids.1)

Eadg. 23 wlitig and winsum

Met. $9^{58}$ geond pas lænan worold

Ps. C 104 to pinum willan weordan

B. 1215 heo fore pæm werede spræc | (El. 332)

Jud. 161 Here wæs on lustum

Exod.564 After pam wordum werod wæs on salum

B. 497 pæs wæs hæleða dream

B. 2591, 2845 Næs pa long to pon

B. 2923 ac wæs wide cuð

B. 50 Men ne cunnon

secgan to sote

Cri. 1539 pær hi leahtrum fa lege gebundne

swylt prowiat 
4430 Bithiu gi an hellia sculun tholon an thiustre

2619

2729

2731 So uuas thero liudeo thau

2743 Uuas thes an luston landes hirdi

2778 hie ni mohta is quidi liogan

2807 hobdu bihaunan

2819 antthat an aband seg sunna to sedle

2852 That folc stillo bed, sat gisithi mikil

3421 sumarlanges dages

3579 hren sia thuo mid is handon 3681 sagdun uualdande thanc

3614 mahtig drohtin is selbes suno sendean uuelda

3683 Thuo gisah uualdand Crist blican thena berges uual endi bu Judeono.

4075 fuldu bifolhan

4241 so lioht ostana quam |

4278 Thuo thie rikeo sprak her hebancuning, hordun thia oora

4299 huann thiu maria tid giuuirthit an thesaro uueroldi

4342 ist sumer ginahid uuaram endi uunsam endi uueder sconi

4801 so hie err deda

5060 irri endi enhard

5563 uuerco te lone

5729 Hie giuuet im thuo forth thanan

5914 uuas iro iamar muod

\section{Hildebrand.}

1 Ik gihorta tat seggen

7

er uuas heroro man ferahes frotoro

15 dat sagetun mi usere liuti alte joh frote
B. 588 pæs pu in helle scealt werhðo dreogan

Aepelst. 3 ealdorlangne tir $\mid$

Men. 17 swa hit geterldon geo frode gesioss

An. 177 Swa is pære nenigeo peaw

B. 607 pa wæs on salum sinces brytta (El. 194)

B. 80 He beot ne aleh

Met. $1^{43}$ heafde beheawan

Aepelst.16 oðpæt seo æpele gesceaft sah to setle

B. 301 flota stille bad, [med scip seomode on sole sidfæp-

Kl. 37 sumarlangne dæg (Jul. 495)

Met. $4^{19}$ sumurlange dagas

B. 722 syððan he hire folmum hran

An.147! sægde metude panc (By.147)

Men. 226 fæder engla

his sunu sende

Jud. 136 pæt hie sweotollice geseon mihten

[blican pære wlitegan byrig weallas

Dan. 560 foldan befolen

B. 569 Leoht eastan com ।

By. 91 pa se eorl ... [wæter ongan ceallian pa ofer cald Byrhthelmes bearn, beornas gehlyston

Men. 56 on pam oftust cym seo mære tid mannum to frofre

Met. $11^{\text {B0 }}$ sumor æfter cymeð wearm gewiðeru

B. 1238 swa hie oft ær dydon |

By. 44 yrre and anræd

$\mathrm{Ph} .386$ weorca to lesne

$\mathrm{Ph} .554$ ic gewite ... ponan

B. 50 wæs him geomor sefa, murnende mod.

Pa. 8 We .. hyrdon . . secgan

Rebh. 1 Hyrde ic secgan

By. 317 Ic eom frod feores

B. 415 pa me pæt gelærdon leode $\min \theta$

pa selestan, snotere ceorlas 
21 barn unwahsan

24 friuntlaos man

36 Hadubraht gimalta, Hiltibrantes sunu

42 dat sagetun mi seolidante |

43 dat inan wic furnam ।

47 herron gotan

52 banun ni gifasta

54 breton sinu billiu

54 ti banin werdan

55 ibu dir din ellen taoc।

57 rauba birahanen

60 niuse de motti

64 scarpen scurim

65 staim bort chludun, heuwun harmlicco huitte scilti unti im iro lintun luttilo wurtun

68 giwigan miti wambnum Muspilli.

14 dari is lip ano tod, lioht ano finstri

selida ano sorgun: dar nist siuh neoman

18 pidiu ist durft mibhil daz ze pidenchanne

$20 \mathrm{daz}$ er kotes uuillun kerno kituoe

51 so inprinnant die perga, poum ni kistentit

einic in erdu, aha sar artruknent

muor varsunilhit sih, suilizot longiu

der himil, mano pivallit, prinnit mittilagart

70 daz der man er enti sid upiles kifrumita

81 scal manno gilih fona deru molta arsten

ar dero leuuo vazzon; scal imo avar sin lip piqueman
Gen. 2871 bearn unweaxen

Wand. 45 wineleas guma (An. 1707

freonda leas, B. 1665

winigea leasum)

B. 1383 Beowulf mapelode, bearn Ecgpeowes

B. 37i ponne sægdon sæliðende | Wand. 80, El.131 sume wig fornam |

Deor. 39 holdne hlaford

El. 477 deað orfæstan

Exod. 199 billum abreotan

B. 2203 to banan wurdon ।

An. 460 gif his ellen deah | B. 573 ponne h. e.d.

Rä. $14^{7}$ reafe berofene

B. 1387 wyrce se pe mote

Jud. 78 scearpne mece scurum heardne

Aepelst. 5 bordweall clufon heowan heorolinda hamora lafum

By. 126 wigan mid wæpnum

Cri. 1653 pær is leofra lufu, lif butan endedeaðe, ... hælu butan sare

... dæg butan peostrum ... blis butan sorgum

Cri. 848 Is us pearf micel pæt we . .georne bipencen

Ps. $102^{20}$ pæt hi his willan wyrcean georne

Cri. 965 ponne eal preo on efen nime $\delta$ won fyres wælm

se swearta lig: sæs mid hyra fiscum

eorðan mid hire beorgum and upheofon

torhtne mid his tunglum; teonleg somod [on an pryðum bærneð preo eal

Cri. 1053 pæt hi ær oððe sið worhtun in worulde

Cri. 1030 Sceal ponne anra gehwylc fore Cristes cyme cwic arisan

leotum onfon and lichoman 
90 so dar manno nohhein uuiht pimidan ni mak

93 uuaz er untar mannun mordes kifrumita.

102 augit er dio masun dio er in menniski

duruh desse mancunnes minna

Wessobrunner Gebet.

1 Dat gafregin ih
Cri. 1048 ne magon hord weras heortan gepohtas

for waldende wihte bemiðan

Jud. 181 pe us monna mæst morora gefremede

Cri. 1116 magon him sylfe geseon ponne open orgete pæt he for alda lufan ... .

Dan. 1 Gefrægn ic. Ph. 1 Hæbbe ic gefrugnen.

Die ubereinstimmung im ausdruck zwischen diesen dichtnngen kann auf dreierlei weise herbeigefuhrt worden sein: 1) Es können formeln mit den Angelsachsen vom kontinente in die neue heimat gekommen sein. 2) Einige können nach der angelsächsischen einwanderung tubertragen worden sein. 3) Es können sich angelsächsische phrasen unabhängig von den altsächsischen und althochdeutschen entwickelt haben.

Unter den ersten fall sind wol einige reimformeln einzureihen, welche sich auf kampf beziehen. Krieg war das handwerk der Germanen auf dem festlande gewesen und blieb es in England. Dass mit der sache selbst die auf sie beztiglichen ausdrticke hertlbergenommen wurden, ist begreiflich. Wenn einige phrasen der Angelsachsen ererbt sind, so muss es schon einen gewissen formelvorrat im Westgermanischen gegeben haben. Beweis fur das vorhandensein eines solchen sind die tbereinstimmungen zwischen altsächsischen und althochdeutschen dichtungen:

Wessob. G. 16 enti dinan uuilleon za gauurchanne

Hildebr. 13 al irmindeot

n 54 imo ti banin werdan ।

" 64 scarpen scurim

Musp. 18 pidiu is durft mihhil daz ze pidenchanne allero manno uuelihhemo

23 heizzan lauc

n 35 dar scal er vora rihhe az rahhu stantan

„ 89 dia dar ar resti uf arstent |
Hel. 1589 to giuuirkeanne thinan unilleon

n 1773 al irminthiod

" $644 \mathrm{im}$ ti banen uuerthan ।

" 5136 scarpon scuron

4375 For thiu scal allaro leodo gihuilic gethenkean for them thinge, thest is tharf mikil manno gihuilicon

2573 heta logna

n 1894 Oft sculun gi thar for rikie gibundana standan

n 2201 upp astandan । arisan fan theru restun. 
Musp. 102 dio er in menniski duruh desse mancunnes minna
Hel. 5504 hie it all githolodi thiodo drohtin mahtig thuru thia minnia manno cunnies.

Wie kamen die Germanen zu ihrem phrasenvorrat?

1. Eine anzahl der formeln sind einfach auf rechnung des allgemein epischen stiles zu setzen. Phrasen wie and pat word acwaed, ageaf him andsware, after pissum wordum u. ä. haben ihre entsprechungen in den epischen dichtungen verschiedener völker. Es ist gar nicht auffallend, wenn wir sie bei den germanischen stämmen widerfinden.

2. Bei den Germanen speziell war fur die ausbildung stehender phrasen die alliteration ein wichtiges förderungsmittel. Besonders in der ersten hälfte der alliterierenden verszeile, wo zwei gleiche stäbe verlangt werden, entstehen leicht formelhafte wendungen. Die entwicklung derselben begtinstigen namentlich:

a) Die verbindung von synonymen und die antithetische zergliederung, z. b. eard and eðel, hord and hamas, land and leode, gifre and gradig, habban and healdan, leof and lað, earm and eadig, innan and utan.

b) Die wortkomposition: frece and grim wird der alliteration wegen $\mathrm{zu}$ frece and ferdgrim, fus on weg zu fus on foroweg. Ebenso entstehen ausdrticke wie heedre heofontungol, sunne swegltorht, morgen marelorht, eorde and upheofon, weroda wuldorcyning, sigora sobcyning, heofena heahfrea, lifes lèohtfruma u.a.

c) Der gebrauch von präpositionalen verbindungen wie under wolcnum, under roderum $\mathbf{u}$. dergl. Die wahl des substantivums hängt von dem voransgehenden ersten stab ab. Daher die formeln weras under wolcnum, hole halig under hrusan, men on moldan, eorl on eorðan, rice under roderum, hus under hrofe, geseon under swegle, feol to foldan, lêerde under lyfte, murnan on mode, hycgan on heortan, forht on ferhðe, hrinan mid handum.

In der zweiten halbzeile finden sich besonders häufig:

a) Die sogenannten flicksätze mit swa: swa him god bebead, swa he oft (ar) dyde, swa him gemet waere.

b) Ausrufe, wie baet waes god cyning, naes poet herlic doed $\mathrm{u}$.ä.

Auch eine reihe weitgehender thereinstimmungen sind durch den einfluss der alliteration zu erklären. Durch die ein- 
wirkung der letzteren können ganze situationsschilderuzigen ebenso phrasenhaft werden wie. einfache reimformeln. Es ist von vornherein zu erwarten, dass darstellungen gleicher handlungen und zustände wenigstens der hauptsache nach gleiche ausdrticke enthalten. Letztere mussen als die dem sinne nach wichtigsten wörter den stabreim trágen. Sind nun durch denselben einzelne teile des satzes in ein festes geftge gebracht, so wird sich die ganze konstruktion nicht so loicht ändern. Sie kann erstarren und formelhaft werden. Wenn dann einmal eine bequeme phrase vorhanden ist, so wird nicht jeder dichter, sobald dieselbe situation widerkehrt, immer eine nene schilderung derselben beginnen. Und selbst wenn er dies tun wollte, so würde ihm doch der stabreim bei dem gleichen stoffe auch wider gleiche ausdrticke zuftuhren, die neue schilderung wurde sich von der alten nur wenig, oft vielleicht gar nicht unterscheiden.

Die religiösen formeln können nicht mit vom festlande gekommen sein, da ja die Angelsachsen erst in England mit dem christentume bekannt wurden. Sie mussen also zu jenen phrasen gehören, welche eine spätere thbertragung oder eine selbständige entwicklung auf englischem boden sind. Einige ubereinstimmungen des ausdruckes mögen auf den gegenseitigen einfluss zwischen Angelsachsen und kontinentalen zartlckzufuhren sein. Ob man aber stets mit sicherheit feststellen kann, welche phrasen die ubertragenen sind, scheint zweifelhaft. Man sagt allerdings, jene stellen seien entlehnt, welche bei ganz unpassender gelegenheit angebracht werden, widersprtiche zu fruher gesagtem enthalten $u$. $\ddot{a}$. Allein ich glaube, dass der gebrauch der einzelnen wendungen ein viel zu mechanischer, formelhafter war, als dass man daraus sichere schltusse ziehen durfte. Von einem und demselben autor wird zuweilen in einer dichtung derselbe ausdruck anf versehiedene dinge bezogen. Glöde stellt Anglia IX, s. 308 dar, wie Cynewulf in der Elene die kenning 'sigebeam' sowol fur das kreuz Christi als auch fur die kreuze der schächer gebraucht. Ein weiteres beispiel fur unpassenden gebrauch einer formel fuhrt Sarrazin a. a. o. s. 119 an: Die phrase 'wlitig on wage' wird auf ein altes schwert angewendet. Gewiss nicht passend. ${ }^{1}$ Aber dieser gebrauch erklärt sich leicht dadurch, dass dem dichter die dar-

1 Vgl. dagegen Anglia XI, 537.

R. W. 
stellung eines blinkenden schwertes viel geläufiger ist als die eines alten, ungebrauchten. Er uberträgt ohne weiteres die formel von jenem auf dieses und bildet sich nicht erst immer neue phrasen.

Eine grosse anzahl übereinstimmungen können ganz unabhängig von einander entstanden sein. Es gibt zwar einige parallestellen, welche in der ganzen ags. poesie nur einmal vorkommen, dagegen in den kontinentalen dichtungen sich wider finden, wie By. 89, Aep. 3, B. 237 ff. Wollte man aber hier entlehungên annehmen, so müsste der gegenseitige einfluss von ags. und kontinentaler dichtung ein ungemein grosser gewesen sein. Der dichter des Hel. wüsste B. gekannt haben, und die dichter von By. und Aep. mlissten widerum nach dem muster des Heliand gearbeitet haben. Allein es ist nicht nötig, an eine solche, jahrhunderte lang dauernde wechselseitige einwirkung zu denken. Wenu die einzelnen dialekte einen teil des germanischen formelschatzes geerbt haben, so konnten sie diesen’ später ganz selbständig weiter ausbilden. Dabei können in verschiedenen dialekten oft ähnliche oder gleiche phrasen produziert worden sein. Die ubereinstimmungen zwischen B. 237 ff. und Hel. 554 ff. erklärt sich z. b. dadurch, dass in beiden fällen ähnliche situation vorliegt und diese bringt ähnlichen ausdruck mit sich. Herodes will gleich dem strandwart von fremden ankömmlingen erfahren, woher sie kommen und was der zweck ihrer reise sei. Ihre stattliche erscheinung lässt ihn, wie den wächter in B., vermuten, dass sie nicht von geringer herkunft seien.

Es ist ferner ganz wol denkbar, dass gleiche lateinische ausdrucke in den verschiedenen germanischen dialekten wider gleiche wendungen hervorrufen. Stellen wie: dinan uuilleon $z a$ gauurchanne (Wessobr. G. 16) und: te giuuirkeanne thinan willeon (Hel. 1589); ferner: daz er kotes uuillun kerno tuoe (Musp. 20) und: paet hi his willan wyrcean georne (Ps.10220) können leicht durch dieselbe lateinische formel entstanden sein. Ebenso sind ohne zweifel die zahlreichen tbereinstimmungen zwischen Maspilli und Crist durch verwandte lateinische vorbilder veranlasst worden.

Unter denjenigen parallelstellen, welche in Ags. oft, in der ahd. und as. alliterationspoesie nur selten oder gar nicht begegnen, sind besonders die zu nennen, welche sich auf see- 
fahrt beziehen. Derartige reimformeln und phrasen treffen wir allenthalben in den ags. dichtungen, fast nie im Ahd. und As. Ferner kommen ausfuhrliche schilderungen von kampfscenen im Ags. sehr häufig vor, während die auf krieg beztiglichen phrasen der kontinentalen dichtungen meist bloss allgemeine reimformeln sind. Die darstellung derartiger scenen ist geradezu typisch. Das geschrei der schlachtraben wird immer in gleicher weise erwähnt. Desgleichen wird die schmähliche flucht der feigen stets mit gleichen oder ähnlichen worten dargestellt, z. b. Gen. 1999, Aep. 36, El. 134, Wald. 15 u. ö. - Beim untergang von helden werden oft dieselben begleitenden nebenumstände angeführt. So finden sich viele zitge ans $B$. in By. wider. By. wird von seinen genossen im stiche gelassen wie B. (By. 185, 193 f., B. 2598), nur wenige harren bei ihm aus, darunter besonders ein heldenhafter jüngling (210, vgl. B. 2626). Es wird der von dem herrn empfangenen auszeichnung (By. 196, B. 2606) gedacht, sowie der in der halle gegebenen versprechen (By. 211 ff., B. $2631 \mathrm{ff}$.). Kurz vor seinem tode spricht der held ein dankgebet (By. 173 f., B. 2794 f.).

Aus dem häufigen vorkommen solcher parallelstellen ist weiter gar nichts zu schliessen, als dass sie, dem gewöhnlichen leben der Angelsachsen entnommen, bei denselben allgemein beliebt waren. Infolge ihres fortwährenden gebrauches werden sie phrasenhaft und dann ebenso mitunter an unpassender stelle angebracht wie die einfachen reimformeln. Sarrazin fuhrt a. a. o. s. 120 die rede des Wiglaf als beispiel an. Derartige fälle finden sich auch sonst, dass personen durch erinnerung an ihre fruheren versprechungen zum ausharren und zur thchtigkeit angeregt werden. Ausser B. 2663 und Gâ. 144 noch in B. 2632 , Bo. 13, By. 212.

Was die ausbreitung des ags. phrasenschatzes betrifft, so haben alle dichtungen an demselben anteil, jedoch nicht in gleichem masse. Im allgemeinen weisen die heldenhaften epen die grösste anzahl von parallelstellen aus, ihnen zunächst steht die geistliche epik, schliesslich die didaktik. Im verhältniss zu ihrer verszahl haben z. b. Wand., By. gegen 10\%, Aepelst. $10 \%$, die echt Cynewulfischen dichtungen, Exod., Dan., Sat. etwa $4 \%$, Seel., Sch., Dom., Cræ. nur 1\% parallelstellen.

WIEN. J. KAIL. 